Wirksamkeit von Ustekinumab, einem gegen II-12, IL-23 bzw. den Th17-Signalweg gerichteten monoklonalen Antikörper, bei der Psoriasisarthritis. Forscher aus Irland und den USA haben nun anhand von Serumproben der Studienteilnehmer die prognostische Relevanz verschiedener Biomarker untersucht.

Sie interessierte dabei besonders, welcher Zusammenhang zwischen den Th17-assoziierten Zytokinen und der Krankheitsaktivität besteht, inwiefern Ustekinumab die Zytokinspiegel beeinflusst und inwiefern dies mit dem Therapieansprechen korreliert. Von 927 Teilnehmern der PSUMMIT 1und der PSUMMIT 2-Studie lagen Serumproben zur Biomarkeranalyse vor. Die Forscher werteten dabei sowohl vor als auch während der Ustekinumab-Therapie entnommene Proben aus. Sie bestimmten hierbei die Konzentrationen von IL-17A, IL17F, IL-23 sowie des C-reaktiven Proteins (CRP). Im Rahmen der post hoc-Analyse prüften sie, welcher Zusammenhang zwischen dem Ausgangsbefund dieser Biomarker bzw. den Spiegeln nach 4 und 24 Wochen und der initialen Krankheitsaktivität an Haut und Gelenken bzw. dem Therapieansprechen bestand.

\title{
Ergebnisse
}

Die zu Studienbeginn bestimmten IL-17Aund IL-17F-Konzentrationen - und in geringerem Umfang auch die IL-23-Konzentration - korrelierten positiv mit der kutanen Krankheitsaktivität vor Therapieeinleitung, nicht jedoch mit der muskuloskelettalen Krankheitsaktivität. Die CRP-Konzentration korrelierte zu diesem Zeitpunkt weder mit der Haut- noch der Gelenkaktivität. Ein Zusammenhang zwischen den basalen Zytokin-

\section{Psoriasisarthritis: Sagen Biomarker das Ansprechen auf Ustekinumab voraus?}

\author{
Siebert S et al. Responsiveness of Serum C-Reactive \\ Protein, Interleukin-17A, and Interleukin-17F Levels \\ to Ustekinumab in Psoriatic Arthritis: Lessons From \\ Two Phase III, Multicenter, Double-Blind, Placebo- \\ Controlled Trials. Arthritis Rheumatol 2019; 71: \\ 1660-1669. doi: 10.1002/art.40921
}

Zwei multizentrische, Placebo-kontrollierte Phase III-Studien belegen die spiegeln/dem CRP und dem klinischen Therapieansprechen auf Ustekinumab nach 24 Wochen bestand nicht. Bezüglich der pharmakodynamischen Veränderungen der Serumbiomarker im Behandlungsverlauf zeigte sich: Im Vergleich zu Placebo nahmen unter Ustekinumab die Spiegel von IL-17A, IL-17F und CRP signifikant ab. Anschließend prüften die Forscher den Zusammenhang zwischen diesen Biomarkerveränderungen und dem klinischen Therapieansprechen. Sie fanden folgendes heraus: Mit Ustekinumab behandelte Patienten, bei welchen sich nach 24 Wochen der 
„Psoriasis Area and Severity Index“-Score um 75\% bzw. der Erkrankungsschweregrad gemäß Kriterien des „American College of Rheumatology“ um $20 \%$ verbessert hatten, wiesen im Vergleich zu Nonrespondern eine deutlichere Abnahme des CRP-Spiegels - nicht jedoch der IL-17A- oder IL-17FSpiegel - auf.

FAZIT

Bei der Psoriasisarthritis korrelieren die prätherapeutischen IL-17A-, IL-17F- und IL-23-Spiegel mit der kutanen, nicht jedoch mit der Gelenk-Krankheitsaktivität, schlussfolgern die Autoren. Obwohl unter Ustekinumab die Zytokin- und CRP-Konzentrationen rasch abfallen, ist eine Vorhersage des Therapieansprechens anhand der initialen Spiegel nicht möglich. Zukünftige Studien müssen nun nach weiteren potenziell klinisch relevanten Biomarkern dieser pathophysiologisch komplexen Erkrankung suchen.

Dr. med. Judith Lorenz, Künzell 